\title{
HOW MANY KNOTS HAVE THE SAME GROUP?
}

\author{
JONATHAN SIMON ${ }^{1}$
}

\begin{abstract}
Let $K$ be a knot in $S^{3}, G=\pi_{1}\left(S^{3}-K\right), n=$ number of prime factors of $K, \nu(G)=$ number of topologically different knot-complements with group $G$ and $\kappa(G)=$ number of distinct knot types with group $G$.

THEOREM. If $K$ is prime, then $\nu(G)<2$. If $n>2$, then $\nu(G)=\kappa(G)<2^{n-1}$. For each $n>2$, the bound $2^{n-1}$ is the best possible. For $K$ prime, we still have the conjecture $\nu(G)=\kappa(G)=1$. If $K$ is a cable-knot, then $\kappa(G)<2$.
\end{abstract}

Let $K$ be a tame knot in the 3-sphere $S^{3}$ and let $G$ denote $\pi_{1}\left(S^{3}-K\right)$. If $L$ is a knot whose group is isomorphic to $G$, must $K$ and $L$ be equivalent? Must the complements of $K$ and $L$ be homeomorphic? How many mutually inequivalent knots, or mutually nonhomeomorphic knot-complements, can have the same group? These problems are discussed in [8], [5, Problems 1.13 and 1.15] and, most recently, [3], which is the basis for this paper.

W. Thurston has announced a proof [10], using hyperbolic structures, that for knots whose exteriors have no essential annuli or tori, there are at most finitely many knots (the number possibly varying with $K$ ) having a given group. For such knots (in fact, for all knots whose exteriors have no essential annuli) the groups determine the complements [2], so Thurston really is dealing with the question of how well the complements determine the knots.

In [8], we gave an elementary proof that if certain prime knots that are not hyperbolic, namely cable knots, also have the property that groups determine complements, then at most three mutually inequivalent knots in $S^{3}$ can have the same complement. In this paper, we show (Corollary 2) that if $K$ is any prime knot, then there are at most two $\left(S^{3}-K\right.$ and perhaps one other) knot-complements with group $G$. The case where $K$ is a cable knot is the only one (see any of the following: [3]; [5, Problem 1.13] or [8, Paragraph 4]) for which we cannot (yet?) prove that the complement of a prime knot is determined by its group. But we can prove (Theorem 1) that there are at most two cable knots (hence, at most two cable knot-complements) having a given group.

Certain composite knots are the only known examples of inequivalent knots with isomorphic groups, the archetypes being the square knot and the granny knot [4], [7]. It is shown in [3] that composite knots with isomorphic groups actually are composites of equivalent knots and, consequently, at most finitely many composite

Received by the editors May 22, 1978 and, in revised form, April 9, 1979.

AMS (MOS) subject classifications (1970). Primary 55A25, 57A10.

${ }^{1}$ Research supported by University of Iowa Developmental Assignment, National Research Council of Canada Grants A-5614, A-5602, and A-8207, NSF Grant MCS76-06992 and the hospitality of York University. 
knots can have a given group. By slightly sharpening the observations made in [3], we can show (Theorem 3$)$ that if $K$ is a composite with $n$ prime factors $(n>2)$ then $G$ is the group of at most $2^{n-1}$ knots. By composing invertible, nonamphicheiral knots (e.g. torus knots), it may be seen that the bound $2^{n-1}$ actually is attained for each $n$.

Definitions and notation. Knots $K, L$ in $S^{3}$ are equivalent if there exists a homeomorphism $h: S^{3} \rightarrow S^{3}$ such that $h(K)=L$. The closed complement of a regular neighborhood of $K$, sometimes called the exterior of $K$, is denoted $C^{3}(K)$. There are, up to isotopy, unique simple closed curves $\mu$ and $\lambda$ on $\partial C^{3}(K)$ such that $\mu \perp \lambda, \mu$ bounds a disk pierced once by $K$ and $\lambda$ is null-homologous in $C^{3}(K)$. When we need to be precise about orientations, we adopt the conventions that $K$ is an oriented curve in a right-hand oriented $S^{3}$, the longitude $\lambda$ is oriented parallel to $K$ and the meridian $\mu$ is oriented so that its linking number with $K$ is +1 . The equation $J=(p, q ; K)$ means that $J$ is a simple closed curve on $\partial C^{3}(K)$ and $J$ is oriented so that, as 1-cycles on $\partial C^{3}(K), J$ is homologous to $p \mu+q \lambda$. The statement $J$ is a $(p, q)$-cable about $K$ means that for some orientations of $\mu$ and $\lambda$, $J=(p, q ; K)$. When $|q| \geqslant 2$, we call $J$ a cable-knot with core $K$. If $K$ is an oriented knot then the inverse of $K$, denoted $K^{-1}$, is the same knot with its orientation reversed. The mirror-image of $K$, denoted $K^{*}$, is the image of $K$ under a reflection of $S^{3}$.

\section{Prime knots.}

THEOREM 1. At most two inequivalent cable knots have the same group, that is, if $K_{0}, K_{1}, K_{2}$ are cable knots with isomorphic groups, then two (or all) of the knots are equivalent.

Proof. Suppose $K_{0}=\left(p, q ; H_{0}\right)$. If $H_{0}$ is unknotted, then $K_{0}$ is a torus knot ([1] or [9]) and $K_{1}, K_{2}$ are equivalent to $K_{0}$ [6]. Assume from now on that $H_{0}$ is knotted.

$\$ 3$ of [3] provides us with the following information. For $i=1,2, K_{i}=$ $\left(p_{i}, q_{i} ; H_{i}\right)$ where $\left|p_{i}\right|=|p|,\left|q_{i}\right|=|q|$ and the exteriors of $H_{i}$ and $H_{0}$ are homeomorphic. For $i=0,1,2$, the manifold $C^{3}\left(K_{i}\right)$ is cut by an essential annulus $A_{i}$ into a knot-manifold $C^{3}\left(H_{i}\right)$ and a solid torus, and $K_{i}$ is parallel in $S^{3}$ to a component of $\partial A_{i}$. Finally, there are homotopy equivalences $F_{i j}: C^{3}\left(K_{i}\right) \rightarrow C^{3}\left(K_{j}\right)(i=0,1, j$ $=1,2)$ such that $F_{i j}$ maps $\left(C^{3}\left(H_{i}\right), A_{i}\right)$ homeomorphically onto $\left(C^{3}\left(H_{j}\right), A_{j}\right)$. Let $\tilde{F}_{i j}$ denote the restriction of $F_{i j}$ to $C^{3}\left(H_{i}\right)$. To show that some of $K_{0}, K_{1}, K_{2}$ are equivalent, it suffices to show that one of the homeomorphisms $\tilde{F}_{01}$, $\tilde{F}_{12}$, or $\tilde{F}_{02}=\tilde{F}_{12} \circ \tilde{F}_{01}$ extends to an autohomeomorphism of $S^{3}$.

Let $\mu_{i}, \lambda_{i}$ be a meridian and longitude for $H_{i}(i=0,1,2)$. Orient $\mu_{0}, \lambda_{0}$ standardly, and orient $\mu_{i}, \lambda_{i}(i=1,2)$ so that the components of $\partial A_{i}$ are homologous to $p \mu_{i}+q \lambda_{i}$ on $\partial C^{3}\left(H_{i}\right)$. There exist numbers $\alpha, \beta, \gamma, \delta, \varepsilon, \eta$, each of absolute value 1 , and integers $x, y$ such that the actions of $\tilde{F}_{i j}$ are as follows (the third line comes from the fact that $\tilde{F}_{i j}$ maps $A_{i}$ homeomorphically onto $A_{j}$ ). 


\begin{tabular}{lll}
$\tilde{F}_{01}$ & $\tilde{F}_{12}$ & $\tilde{F}_{02}$ \\
\hline$\mu_{0} \rightarrow \mu_{1}^{\alpha} \lambda_{1}^{x}$ & $\mu_{1} \rightarrow \mu_{2}^{\gamma} \lambda_{2}^{y}$ & $\mu_{0} \rightarrow \mu_{2}^{\alpha \gamma} \lambda_{2}^{\alpha y+\delta x}$ \\
$\lambda_{0} \rightarrow \lambda_{1}^{\beta}$ & $\lambda_{1} \rightarrow \lambda_{2}^{\delta}$ & $\lambda_{0} \rightarrow \lambda_{2}^{\beta \delta}$ \\
$\mu_{0}^{p} \lambda_{0}^{q} \rightarrow\left(\mu_{1}^{p} \lambda_{1}^{q}\right)^{\varepsilon}$ & $\mu_{1}^{p} \lambda_{1}^{q} \rightarrow\left(\mu_{2}^{p} \lambda_{2}^{q}\right)^{\eta}$ & $\mu_{0}^{p} \lambda_{0}^{q} \rightarrow\left(\mu_{2}^{p} \lambda_{2}^{q}\right)^{e \eta}$
\end{tabular}

We shall show now that at least one of the exponents $x, y, \alpha y+\delta x$ must equal 0 , so the appropriate $\tilde{F}_{i j}$ extends to $S^{3}$. Assume $x \neq 0$ and $y \neq 0$. If we recompute $\tilde{F}_{01}\left(\mu_{0}^{p} \lambda_{0}^{q}\right)$ in terms of $\tilde{F}_{01}\left(\mu_{0}\right)$ and $\tilde{F}_{01}\left(\lambda_{0}\right)$, we see that $\alpha=\varepsilon$ (since $p \neq 0$ ) and $p x=q(\alpha-\beta)$. Since $p \neq 0, x \neq 0$, and $|\alpha|=|\beta|=1$, we conclude $\beta=-\alpha$ and $x=2 \alpha q / p$. Similarly, we have $\delta=-\gamma$ and $y=2 \gamma q / p$. Thus $\alpha y+\delta x=$ $(2 q / p)(\alpha \gamma+\delta \alpha)=0$.

\section{COROLlaRY 2. At most two prime knot-complements can have the same group.}

Proof. Suppose $K_{0}$ is a prime knot and $K_{1}, K_{2}$ are knots whose groups are isomorphic to $\pi_{1}\left(C^{3}\left(K_{0}\right)\right)$. As discussed in [3], [5, Problem 1.13] or [8, Paragraph 4], we can conclude that all the $C^{3}\left(K_{i}\right)$ are homeomorphic except perhaps in the case where $K_{0}$ is a cable knot. In this case, Theorem 1 applies.

Remark. One might ask whether it is possible to construct the unique (by Corollary 2) candidate for another prime knot-complement having the same group as a given one. What we can construct is a knot in a homology 3-sphere with knot group isomorphic to the given one and knot-complement, in general, not homeomorphic to the given one. The conjecture that prime knot-complements are determined by their groups translates into the conjecture that the homology spheres obtained are never homeomorphic to $S^{3}$. Corollary 2 translates into the fact that for each knot $K$ there is at most one homology sphere we need to test. The algorithm for testing a particular knot $K_{0}$ is as follows. If $K_{0}$ is not a cable knot, then $C^{3}\left(K_{0}\right)$ is determined, among knot manifolds in $S^{3}$, by its group. If $K_{0}=$ $\left(p, q ; H_{0}\right)$ (here we are being careful about orientations) and $|p| \geqslant 3$ then, again, $C^{3}\left(K_{0}\right)$ is characterized by its group [3]. If $|p| \leqslant 2$ then replace $H_{0}$ by whichever of the four knots $H_{0}, H_{0}^{-1}, H_{0}^{*}$, or $H_{0}^{*-1}$ that enables us to write (using standard orientations) $K_{0}=\left(|p|,|q| ; H_{0}\right)$. If $K_{0}=\left(1,|q| ; H_{0}\right)$, (try to) show that the surgery manifold $M^{3}\left(H_{0} ; 1,2 q\right)$ is not $S^{3}$; if $K_{0}=\left(2,|q| ; H_{0}\right)$, show $M^{3}\left(H_{0} ; 1, q\right)$ is not $S^{3}$.

2. Composite knots. The square and granny knots are the best known examples of inequivalent composite knots with the same group. The granny is the sum, $K \# K$, of two identical trefoil knots. The square knot is the sum, $K \# K^{*}$, of a trefoil and its mirror image. The reason the knots are different is intuitively clear because $K$ and $K^{*}$ are somehow different. The reason the groups are isomorphic is that $K$ is invertible! To establish Theorem 3 below, we combine the latter insight, for which some thanks are due to D. R. McMillan, with results of [3].

The operation of composition of knots is well defined on oriented isotopy types but not well defined on knot types. We can ease this confusion by defining knots as 
purely algebraic objects. Because of [11], we can define an oriented knot $K$ to be a triple $(G, \mu, \lambda)$, where $G$ is isomorphic to $\pi_{1}\left(S^{3}-K\right)$ and $\mu$ and $\lambda$ are elements of $G$ corresponding to standardly oriented meridian and longitude of $K$. Knots $K_{1}$ and $K_{2}$ are of the same oriented isotopy type if and only if there exists an isomorphism of triples $\left(G_{1}, \mu_{1}, \lambda_{1}\right) \simeq\left(G_{2}, \mu_{2}, \lambda_{2}\right)$. The composition $K_{1} \# K_{2}$ is defined to be $\left(G_{1} \mu_{1}^{*}=\mu_{2} G_{2}, \mu_{1}, \lambda_{1} \lambda_{2}\right)$. We can define an automorphism $\theta$ of $G_{1} \underset{\mu_{1}=\mu_{2}}{*} G_{2}$ by $\theta(g)=\lambda_{2} g \lambda_{2}^{-1}$. Since $\mu_{2}$ commutes with $\lambda_{2}$ and $\mu_{1}=\mu_{2}, \theta$ defines an isomorphism between the triples representing $K_{1} \# K_{2}$ and $K_{2} \# K_{1}$. This is an alternate proof that composition of knots is commutative. If $K=(G, \mu, \lambda)$ then $K^{-1}=$ $\left(G, \mu^{-1}, \lambda^{-1}\right)$ and $K^{*}=\left(G, \mu^{-1}, \lambda\right)$. The knot $K$ is invertible if and only if $(G, \mu, \lambda) \simeq\left(G, \mu^{-1}, \lambda^{-1}\right)$ and amphicheiral if and only if $(G, \mu, \lambda) \simeq\left(G, \mu^{\varepsilon}, \lambda^{-\varepsilon}\right)$ for some $\varepsilon= \pm 1$. The composition $K_{1} \# K_{2}^{*}=\left(G_{1}, \mu_{1}, \lambda_{1}\right) \#\left(G_{2}, \mu_{2}^{-1}, \lambda_{2}\right)=$ $\left(G_{1_{\mu_{1}-\mu_{2}-1}^{*}} G_{2}, \mu_{1}, \lambda_{1} \lambda_{2}\right)$. Comparing this with $K_{1} \# K_{2}$, we see that the nicest situation in which the composite knot groups will be isomorphic is when $G_{2}$ admits an automorphism $\phi$ such that $\phi\left(\mu_{2}\right)=\mu_{2}^{-1}$. If $\phi$ comes from a symmetry of the knot $K_{2}$, then $\phi\left(\lambda_{2}\right)=\lambda_{2}^{ \pm 1}$. If $\phi\left(\lambda_{2}\right)=\lambda_{2}$ (the case where $K_{2}$ is amphicheiral) then $\phi$ defines an equivalence between $K_{1} \# K_{2}^{*}$ and $K_{1} \# K_{2}$. If $\phi\left(\lambda_{2}\right)=\lambda_{2}^{-1}$ (the case where $K$ is invertible) we have the square vs. granny situation of isomorphic groups but apparently inequivalent knots.

THEOREM 3. If $G$ is the group of a knot with $n$ prime factors $(n \geqslant 2)$, then $G$ is the group of at most $2^{n-1}$ mutually inequivalent knots.

REMARK. If prime knots are indeed determined by their groups, then the hypothesis $n \geqslant 2$ is unnecessary and Theorem 3 is the desired answer to the title of this paper.

Proof of Theorem 3. Let

$$
K=K_{1} \# \cdots \# K_{n}=\left(G_{1}, \mu_{1}, \lambda_{1}\right) \# \cdots \#\left(G_{n}, \mu_{n}, \lambda_{n}\right)
$$

and suppose $L$ is a knot whose group is isomorphic to the group of $K$. The statement of Theorem 1 of [3] implies that

$$
L=\left(G_{1}, \mu_{1}^{\varepsilon_{1}}, \lambda_{1}^{\delta_{1}}\right) \# \cdots \#\left(G_{n}, \mu^{\varepsilon_{n}}, \lambda^{\delta_{n}}\right)
$$

where $\left|\varepsilon_{i}\right|=\left|\delta_{i}\right|= \pm 1$, but in the proof of that theorem, it is shown that all the $\varepsilon_{i}$ are equal, since the fact that the preferred meridians of the $K_{i}$ are homologous in $C^{3}(K)$ implies that their images are homologous in $C^{3}(L)$. Thus for some $\varepsilon= \pm 1$,

$$
\begin{aligned}
L & =\left(G_{1}, \mu_{1}^{\varepsilon}, \lambda_{1}^{\delta_{1}}\right) \# \cdots \#\left(G_{n}, \mu_{n}^{\varepsilon}, \lambda_{n}^{\delta_{n}}\right) \\
& =\left(\begin{array}{c}
\left.G_{1} * \cdots * G_{n}, \mu_{1}^{\varepsilon}, \lambda_{1}^{\delta_{1}} \lambda_{2}^{\delta_{2}} \cdots \lambda_{n}^{\delta_{n}}\right) . \\
\mu_{1}^{\varepsilon}=\cdots=\mu_{n}^{e}
\end{array}\right.
\end{aligned}
$$

We now have $2^{n+1}$ choices for $L$, corresponding to choices of $\varepsilon, \delta_{1}, \ldots, \delta_{n}$. Therefore $L$ represents one of $2^{n+1}$ oriented isotopy types and $\frac{1}{4}\left(2^{n+1}\right)=2^{n-1}$ knot types. 


\section{REFERENCES}

1. G. Burde and H. Zieschang, Eine Kennzeichnung der Torusknoten, Math. Ann. 167 (1966), 169-176.

2. C. D. Feustel, On the torus theorem and its applications, Trans. Amer. Math. Soc. 217 (1976), 1-43.

3. C. D. Feustel and W. Whitten, Groups and complements of knots, Canad. J. Math. 30 (1978), 1284-1295.

4. R. H. Fox, On the complementary domains of a certain pair of inequivalent knots, Nedrl. Akad. Wetensch. Proc. Ser. A 55 (1952), 37-40.

5. R. Kirby, Problems in low dimensional manifold theory, Proc. Sympos. Pure Math., vol. 32, part 2, Amer. Math. Soc., Providence, R. I., 1978, pp. 273-312.

6. H. Seifert, Topologie Dreidimensionaler Gefaserter Räume, Acta Math. 60 (1933), 147-238.

7. __ Verschlingungsinvarianten, S.-B. Preuss. Akad. Wiss. 26 (1933), 811-828.

8. J. Simon, On the problems of determining knots by their complements and knot complements by their groups, Proc. Amer. Math. Soc. 57 (1976), 140-142.

9. , Roots and centralizers of peripheral elements in knot groups, Math. Ann. 222 (1976), 205-209.

10. W. Thurston, Proc. Conf. Topology of Manifolds (Univ. of Georgia, Athens, Georgia, August 1977) (to appear).

11. F. Waldhausen, On irreducible 3-manifolds which are sufficiently large, Ann. of Math. (2) 87 (1968), 56-88.

Department of Mathematics, University of Iowa, Iowa City, Iowa 52240 (Current address)

Department of Mathematics, York University, Downsview, Ontario M3J 1P3, Canada 